\title{
A Decomposition of Empirical Distributions with Applications to the Valuation of Derivative Assets
}

\author{
Mondher Bellalah \\ Université de Cergy-Pontoise, France \\ Marc Lavielle \\ Université Paris-Sud, France
}

The selection of an appropriate parameterization of data is a fundamental step in a majority of empirical research effort. Likewise, detecting or estimating features of non-stationarities in data sequences is a critical point in conducting credible research that uses data for inference. In this spirit, this paper presents a simple decomposition of the empirical return distributions of financial assets into the sum of various normal distributions. The decomposition is motivated by the fact that market participants expect distributions to be drawn from two or three possible scenarios. It is also motivated by the recent applications of the EM algorithm to financial data. A parametric and a nonparametric approach are proposed and applied to the empirical distribution of the CAC 40 index traded in the Paris Bourse. We estimate the parameters of the mixture and propose a decomposition into three Gaussian distributions which essentially differ by their variances. The decomposition fits the observed distribution.

An alternative approach, which consists in detecting these changes and estimating the distribution of the returns between two changes is developed. The results are obtained using a segmentation method, which is applied to financial data. One of the main findings in this paper is that the two approaches show the same results and give support to the proposed decomposition. There exists three kinds of regimes in the Paris Bourse and the series of the returns jump from a regime to another one at some random instants. This work might be applied to other data sets or other data generating conditions. It can used for the valuation of standard and exotic derivatives (JEL G1 0, G12, G13).

Keywords: derivatives, distributions, EM algorithm, mixture.

*The author would like to extend his appreciation to the three anonymous referees and the editor, for comments on an earlier draft of this article, which has greatly improved the article.

(Multinational Finance Journal, 2002, vol. 6, no. 2, pp. 99-130)

CMultinational Finance Society, a nonprofit corporation. All rights reserved.

DOI: $10.17578 / 6-2-2$ 


\section{Introduction}

The observed returns distributions of financial assets are central elements in the pricing of securities. On the one hand, models of capital market equilibrium study the equilibrium structure of asset prices and its connection with empirical anomalies. On the other hand, option pricing models show empirical biases as the strike price bias or the smile effect. Since pricing models based on the log-normality assumption reveal several biases, the use of a mixture of normal distributions might help to reduce some of them. The mixture assumption was used by Ritchey (1990) to derive European options prices when the probability density function (PDF) is a mixture of lognormals. Melick and Thomas (1997) estimate the distribution for crude oil during the Gulf crisis and show that it differs from those recovered using standard techniques. The approach used in Melick and Thomas (1997) gives an intuition to our decomposition. They find that the shape of the distribution in the tails depend importantly on the functional form assumed for the distribution and illustrate this point with three observationally equivalent distributions. Their results show that the fitted values from a mixture of three lognormal distributions (MLN) and a single lognormal (SLN) explain the pricing errors of SLN. The converse is not true since the data and MLN reject SLN, but MLN can not be rejected. Lekkos (1999) estimates the probability densities of interest rates using the kernel density estimation method in Ait-Sahalia (1996). This method allows one to identify the "true" non-parametric density function of spot interest rates. The density estimates reveal that there is no standard univariate distribution and there is evidence of multimodality.

The first purpose of this paper is to provide a simple decomposition of the observed distributions into standard normal distributions using alternative approaches. Mixture distributions have received a particular attention in the statistical literature, because of the large number of applications (see Titterington et al. [1985] and Everitt and Hand [1981]). Many authors considered the problem of estimating the order and the parameters of the mixture (see Basford and McLachlan [1985] and Leroux [1992]). The well known Expectation Maximization algorithm, (EM algorithm) proposed by Dempster, Laird and Rubin (1977) can be used for computing the maximum likelihood estimate of the means and variances of a Gaussian mixture (see Redner and Walker, [1984]). For different numbers of components the EM 
algorithm can be applied in the estimation of the marginal distribution of the returns. For the sample used in our study, it turns out that the use of three normal distributions fits very well the empirical marginal distribution of the returns. It is well-known in stock markets that rare economic events like bad news can generate sudden falls in stock prices and the possibility of an offer in the market of corporate control can produce higher stock prices. Therefore, market participants expect distributions to be drawn from some possibilities. This is the main difference with respect to the interest rate market since interest rate is not a tradeable asset. It is expected that the possibility of three regimes in the stock market can lead to a mixture of three distributions rather than two distributions. A mixture of three normal distributions is expected to provide a better fit to the data than the standard normal or lognormal distributions. Here, the data is a time-series, and the time dependence should appear in the model. A widely used approach consists in assuming that the observations follow a mixture model with Markov regime (see Leroux [1992], Ryden et al. [1997]). In that context, the main problem consists in recovering the Hidden Markov Chain that indicates in which state the Chain is at any instant.

We shall adopt another approach, which consists in detecting the sudden changes of regime, and in locating their positions. A wide literature is dedicated to the detection of abrupt changes in a time-series (see Yao [1988], Basseville and Nikiforov [1993], Lavielle and Moulines [1999], Lavielle [1999]). In our context, this method can be useful for financial analysts in portfolio selection and asset's analysis because the location of changes is an important piece of information in portfolio management.

An application of the procedure for change-point detection proposed by Lavielle $(1998,1999)$ to our data set confirms that the returns in the financial market follow three regimes. These three regimes correspond to the three normal distributions estimated in the market place.

We develop a method which provides a simple decomposition of the observed distributions of securities prices into standard normal distribution and propose a method that can detect the changes in the means and variances of the distributions. The decomposition into standard normal distributions allows us to get as close as possible to the observed empirical distribution. For illustrative purposes, the CAC 40 data are used but the method can be applied to other market data. In particular, it can be used in the VaR calculation improving the methodology proposed by JP Morgan in RiskMetrics. In fact, the 
standard calculations of $\mathrm{VaR}$ for portfolios of assets are based on the normal or lognormal assumptions while it is well known that assets returns are multimodal. The sample used comprises CAC 40 index prices and is well explained by a mixture of three normal distributions. It turns out that the returns in the market place follow three regimes, corresponding to three normal distributions with different characteristics. We find that the estimated distributions are consistent with the market reaction to information, in that they reflect the probabilities of major disruption. Our methodology for the detection of changes in the means and variances of the distributions is new and has not been often applied in the financial literature. Some intuitive explanations are given for the observed mixture of normal distributions.

We propose also algorithms that facilitate a decomposition and allow an exact fit to the observed distribution.

The structure of the paper is as follows. Section 2 poses the problem of choosing between several distributions in the modeling of securities returns. This allows us to justify the use of empirical distributions as a starting point in the search for an appropriate distribution for financial assets returns. Section 3 presents a simple decomposition of the distribution of returns into normal distributions. Using the returns on the CAC 40 index, two procedures are proposed. The first is based on a nonparametric estimation. The second approach uses a parametric estimation. Both approaches provide the same result and support the decomposition. Since this question is important for financial research, we address the issue both non parametrically by using a kernel estimator and parametrically under a mixture of normals hypothesis. The nonparametric approach seems to dominate the parametric approach. We develop a procedure for the detection of changes in the distribution of returns and compare our results with the existing literature. Section 4 suggests an application of this method for the valuation of derivative assets. ${ }^{1}$

\section{Characterizing Empirical Distributions}

Models based on frictionless markets and complete information might be inadequate to capture the complexity of action in financial markets. Some factors and constraints, like transaction costs, information costs,

1. The authors thank the referee for suggesting this application. 
restrictions on short sales, entry into the dealer business, the probability attached to a merger or an acquisition, among other factors, may influence the short run behavior of security prices (see Bellalah [1999 $a, b, 2000])$. However, most models developed in financial economics do not explicitly account for these factors, nor their effects on the distributions of securities prices. Therefore, the log-normal assumptions are often used for the dynamics of financial assets. These factors can lead to multimodal distributions and play a central role in capital markets, by affecting the distributions of returns. Market participants use different models when pricing, hedging and managing risks in their portfolios. They spend huge amounts of money to implement new models that "stick" to market prices. This facilitates the management of financial risks contracted by individuals and financial institutions at the micro-structure and macro-structure level. For example, since the price of a derivative security depends on the dynamics of its underlying asset, its expected rate of return might depend on the factors corresponding to market imperfections. When integrated into theoretical pricing models, these factors considered as market imperfections have the potential for explaining reported observed biases in standard models. Unfortunately, some mathematical difficulties and the lack of an economic rationale in modeling the complex financial system prevent the financial economist from elaborating a model that "fits" the observed behavior of financial assets prices. Therefore, a decomposition of observed multimodal distributions into normal distributions might be useful in dealing with financial risks and risk management. This offers a starting point that allows the derivation of financial models which account for the presence of skewness and kurtosis in the distribution of financial returns.

The EM-algorithm has been one of the most successful methods of estimating when the data under study are incomplete. It has been applied to resolve missing data problems involving financial time series. Also, since the second quarter 1996, RiskMetrics monitor introduced the normal mixture model of returns that was found to more effectively measure the tails of selected return distributions. This allows for a larger probability of observing very large returns than the conditional normal distribution.

Many professionals think that the $V a R$ number (obtained from internal VaR models or the models provided by JP Morgan) can be used to aid managers in the understanding of their risk position. In practice, the management of an individual trader's book position requires more 
careful considerations of the risk parameter sensitivities than the single VaR number. This is important for the management of the option price sensitivities or "Greek" letters. However, the models used for the pricing of derivatives do not account for the non-normal features of the distributions of returns. According to Choudry (1996), market crashes and options pose essentially the same problem when calculating $\mathrm{VaR}$. Recall that in the original form of JP Morgan's RiskMetrics, the returns on the underlying portfolio are normally distributed. In this context, $90 \%$ of the returns fall within more or less 1.65 standard deviations (the 5 th and 95th percentiles) of the mean return. The $V a R$ gives in this setting the maximum probable loss on a portfolio under market conditions. One of the methods to overcome the normality assumption and to account for real world distributions is to resort to stress testing and scenario analysis. When the effects of gamma risk (the derivative of the delta) are included in the calculation, the portfolio returns become asymmetric or skewed. In this case, it is possible to include the effects of the gamma for example by calculating the $V a R$ on the basis of a skewed distribution of returns using one of the three following solutions. The first considers an approximation of the skewed distribution by a deformed normal distribution. The second needs the calculation of the 5th and 95 th percentiles from the skewed distribution.

The third is to try to fit the skewed distribution to a more general family of distributions with known statistical characteristic measures. To circumvent these difficulties, it is more interesting to integrate implicitly these considerations in the valuation process of financial assets and derivatives. This can be done by searching for mixed distributions. Lekkos (1999) uses interest rate data and shows that the component distributions were not distinct enough. For this reason, he uses the EM algorithm and assumes that the components exist in a fixed proportion in the mixture of distributions. His study shows that the mixture of two normal distributions provides a better fit to the data than the standard normal or the lognormal distribution. In reality, the work on the mixture-of-normal hypothesis is not new and goes back a long time, e.g., the work of Press (1973), Blattberg and Gonedes (1974) and Kon (1984) among others. The related work on subordinated process modeling can also be traced to the seminal paper of Clark (1973). Two common approaches are often used in the financial literature to study the distribution of securities returns. The first approach describes the stochastic processes that give rise to the returns corresponding to a given distribution. Work in this area include Epps and Epps (1976), 
Tauchen and Pitts (1983), Samorodnitsky and Taqqu (1994) among others. The second approach tries to represent in a usable form a distribution function which fits empirically the observed return distribution in a given financial market. This line of research is used in Fama (1963), (1965) and Mandelbrot (1963) among others. The literature based on mixed distributions uses the first approach. It studies the market process for security prices and puts the accent on the relationship between several market variables as the volume of trading, the price variance, etc. This approach allows sometimes the identification of some well defined distributions. But, when the trading process is studied in detail, it leads to distributions that have no explicit form and hence can not be represented or used in practice. Besides, this literature says nothing about the "shape" of the observed distribution. If this approach is implemented in asset pricing, the main difficulty is the following: how to identify and how to "match" a given stochastic process with an observed empirical distribution?

This question is important because the identification of a given stochastic process that "matches" the observed distribution allows one to build pricing models which give prices that fit market prices. This might allow the elimination of empirical anomalies observed when testing pricing models.

In the second approach by Mandelbrot (1963), the accent is put on the fat tails and the peak of the distribution of returns. It is often observed that there are thicker or thinner right or left tails and a higher peak than the log-normal distribution. However, no study explains why the distribution takes one form or an other and why there are "deviations" with respect to the log-normal distribution. The main distributions reported in the literature in this context are the symmetric stable distributions. These distributions "fit" the observed distributions and show some interesting properties. However, it appears that they remain until (1987) only as an empirical description of the fitted distributions. They present a generalized distribution for describing security price returns: the generalized beta of the second kind, referred to as the GB2. This distribution can be interpreted as a mixed distribution. Bookstaber and McDonald (1987) conclude that the hypotheses of a mixed distribution merits further investigation. Since then, several papers were published on this subject but none offers a simple decomposition of the empirical distributions. Besides, several problems are encountered for giving analytic expressions or estimating the distribution parameters. Longin (1996) studies the asymptotic 
distribution of only extreme stock market returns (minimal and maximal returns). He determines the degree of fatness of the distribution and finds that the mean and variance are finite but the skewness, the kurtosis and all higher moments may be infinite. When these higher moments are infinite, this poses a difficult problem to the researcher who intends to account for these moments in deriving capital asset pricing models or option pricing models. Other studies on Banking supervision, regulation and bankruptcies use the extreme value theory where risk is measured by extreme value statistics rather than the usual measure of variance (see Longin [1996] and Dimson and Marsh [1995]).

The changing character of volatility over time is an important feature in the study of financial returns. Since some years, the emphasis has shifted towards the use of asset pricing models where agents' decisions are based on the distribution of returns conditional on the available information. Researchers account for the time variation in first and second moments of returns since the work of Engle's (1982) on Autoregressive Conditional Heteroscedasticity (ARCH) and Bollerslev's (1986) Generalized ARCH (GARCH). Several applications and variations of these models can be found in Engle (1987), and Koutmos, Negakis and Theodossiou (1993) among others.

The EM algorithm is used for conditionally heteroskedastic factor models in Demos and Sentana (1998). Why not taking directly the empirical distribution and using simple decompositions based on normal distributions? Some studies adopted this line of research which is highly relevant since this problem has been vexing researchers for a long time.(Ritchey [1990], Melick and Thomas [1997] and Lekkos [1999]). Our methodology gives an answer to the above question by adopting two approaches: a nonparametric approach and a parametric approach. The results are confirmed using a segmentation method.

\section{The Decomposition of Empirical Distributions and the Detection of Changes}

This section develops two methods for the decomposition of the distribution of returns into some normal distributions. The first is a nonparametric approach. The second is a parametric estimation. The decomposition method is done using a data set from the Paris Bourse. We propose a model which allows to detect systematically the sudden changes and which locates their positions. This method allows also the 
estimation of the distribution of the data between the abrupt changes. The analysis gives an answer to the two following questions:

What is the shape of the empirical distribution of the CAC 40 index (or the stocks) traded in the Paris Bourse?

Can we decompose the empirical distribution into normal distributions?

The answers need the use of empirical data regarding the CAC 40 index or the returns of some stocks.

In stock markets, rare economic events like bad news can generate sudden falls in stock prices and the possibility of an offer in the market of corporate control can produce higher stock prices. Therefore, it is expected that the possibility of three regimes in the stock market can lead to a mixture of three distributions. The mixture is expected to provide a good fit to the data. The three-regimes hypothesis is appropriate for stock indexes and stocks because of the possible three outcomes in the market: a normal regime, a sudden fall in asset prices, a sudden increase in the prices of these assets. Market participants feel that prices are likely to be drawn from a tri-modal distribution in which each distribution reflects an equity market event.

The tests are conducted using the daily CAC 40 index from January 1988 to December 1990. The data set comprises the stocks and the CAC 40 values corresponding, to the open, the close, the highest, the lowest and the bids and asks for the futures contract on the CAC 40 index during each trading day as well as the amounts of cash distributions. The CAC 40 index is a market-capitalization weighted index of the forty major French companies listed on the French market (RM) (see Briys, Bellalah et al [1998] and Bellalah [2000 b]). It is important to note that the index is adjusted for stock dividends and splits but not for cash dividends. For this reason, dividends are excluded from the daily return calculations.

A. Non-parametric estimation of the distribution of the CAC 40 index.

The empirical distribution corresponding to the CAC 40 index is displayed in figure 1 in two usual forms: an histogram in figure 1-a, and a continuous density estimate in figure 1-b. In fact, the distribution of the returns is clearly continuous while the histogram presents discontinuities. To overcome that, a smoothed version of the histogram can be proposed as an estimate of the probability density function $f$ of 

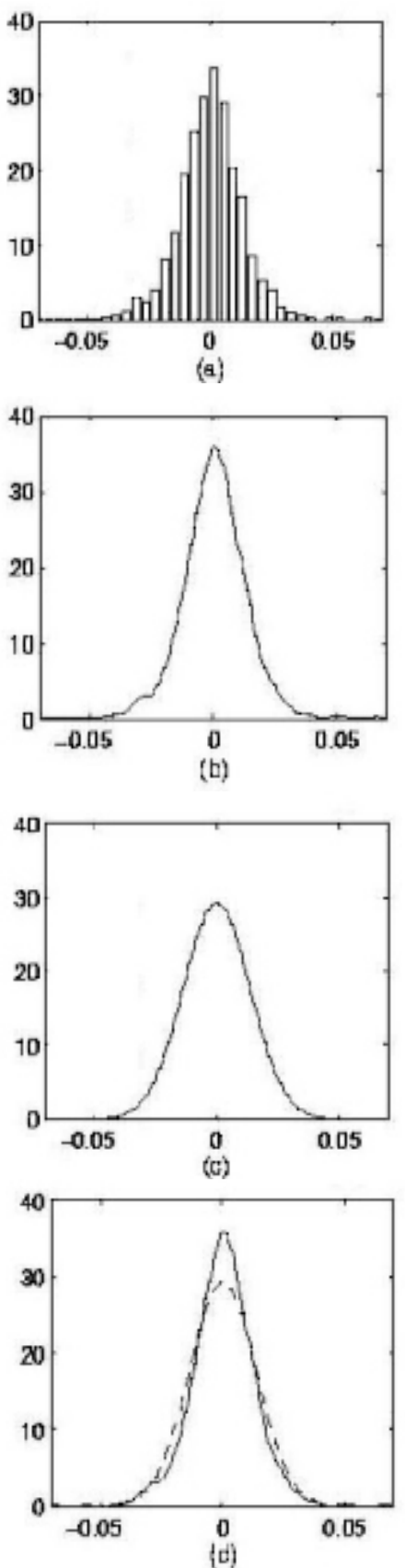

FIGURE 1.-Panels a through d. 
the returns. This kind of estimated is called kernel estimate. Various kernels can be used in this context. In the particular case of a Gaussian kernel, the kernel is defined by:

$$
k(x)=\frac{1}{n} \sum_{i=1}^{n} \frac{1}{\sqrt{2 \pi \sigma^{2}}} e^{-\frac{1}{2 \sigma^{2}}\left(x-x_{1}\right)^{2}},
$$

where $x_{1}, \ldots, x_{n}$ are the observations and $\sigma^{2}$ is a parameter that adjusts the smoothness of $k$. If $\sigma^{2}$ is too small, the estimated function is very rife. On the other hand, some details will disappear with a too big value of $\sigma^{2}$. In our example, we set $\sigma^{2}=4 \times 10^{-6}$.

The empirical distribution seems symmetric around zero. The empirical mean is $\bar{X}=1.13 \times 10^{-4}$, while the empirical variance is $s^{2}=1.86 \times 10^{-4}$. Then, we estimate the standard-deviation of the empirical mean $\bar{X}$ by $s_{X}=\sqrt{s^{2} / n}=3.12 \times 10^{-4}$ (since $n=1908$ in this example). A $90 \%$ confidence interval for the mean of the returns is $[-4$ $\left.\times 10^{-4}, 6.26 \times 10^{-4}\right]$ and consequently, we accept the hypothesis that the returns are centered.

\section{B. Parametric estimation of the distribution of the CAC 40 index}

If we try to adjust a Gaussian distribution to the data, with a mean $\bar{X}$ $=1.13 \times 10^{-4}$ and a variance $s^{2}=1.13 \times 10^{-4}$, the fit would not be perfect. Figure 1-c shows the density of such a distribution and figure 1 -d gives a comparison with the empirical distribution.

Some intuitive reasons might be advanced to explain the observed shapes of the empirical distributions for the CAC 40 index and the empirical distributions of stocks returns in the French market. These reasons may apply as well to the distributions observed in other stock markets. First, since financial assets have always a pay-off which is greater or equal to zero, and assets holders have limited liabilities, the maximum gain from holding a financial asset may be infinite while the maximum loss is restricted to the amount invested in securities. This asymmetric property regarding the gains and losses may justify an asymmetric distribution of asset prices or returns. Second, since the mean return is not a measure of risk, it does not determine the shape of an empirical distribution. Third, since the process of adjustment of expectations regarding the cash-flows of the firms (dividends, coupons, 
and other distributions) is rather "smooth", this produces a different "speed" in the market reaction to information revelation. Hence, there may be a tendency in the market to go up slowly and to fall down suddenly. This case is more pronounced in situations of "panics", financial crashes or mini-crashes where the psychological factor plays an important role, which is not modeled in financial economics. Even if one argues that this explanation is not entirely true, there is a tendency for the volatility to rise when the market falls and to fall when the market rises. These reasons might explain the presence of three possibilities in the market place, which can be reflected in the presence of a mixing of three or more distributions as suggested by the referee.

It is possible to adjust a parametric distribution to the data by assuming that they represent a mixture of Gaussian distributions:

$$
X_{i} \sim \sum_{k=1}^{K} p_{k} N\left(m_{k}, \sigma_{k}^{2}\right)
$$

with

$$
\sum_{k=1}^{X} p_{k}=1
$$

where, $p_{k}$ represents the fraction of the population $k, m_{k}$ its mean and $\sigma_{k}^{2}$ its variance while $K$ is the number of populations. A difficult problem arises at this level. In fact, how can $K$ be chosen and how to estimate the parameters $\theta=\left(p_{1}, m_{1}, \sigma_{1}^{2}, \ldots, p_{K}, m_{K}, \sigma_{X}^{2}\right)$. It is clear that the adjustment of the empirical distribution is better when the number of populations $K$ is high as well as the number of parameters. On the other hand, we have to search for a parametric distribution with a few parameters to be estimated. Therefore, a trade-off must be found between both criteria, i.e; a number $K$ of populations as small as possible which allows at the same time a good adjustment and a good fit to the observations. When $K$ is given, the value of $\theta$ can be estimated by applying the maximum likelihood criteria or by maximizing the likelihood of the observations:

$$
f\left(x_{1}, \ldots, x_{n}, \theta\right)=\prod_{i=1}^{n} \sum_{k=1}^{X} \frac{p_{k}}{\sqrt{2 \pi \sigma_{k}^{2}}} e^{-\frac{1}{2 \sigma_{1}^{2}}\left(x_{1}-m_{1}\right)^{2}} .
$$


TABLE 1. Estimation of the Parameters of a Mixture of Normal Distributions with $K$ Components

\begin{tabular}{ccccc}
\hline$K$ & $k$ & $p_{k}$ & $m_{k}$ & $\sigma_{k}^{2}$ \\
\hline 1 & 1 & 1.00 & $1.1 \times 10^{-4}$ & $1.9 \times 10^{-4}$ \\
2 & 1 & 0.89 & $4.8 \times 10^{-4}$ & $1.2 \times 10^{-4}$ \\
& 2 & 0.11 & $-28.6 \times 10^{-4}$ & $7.3 \times 10^{-4}$ \\
3 & 1 & 0.54 & $-3.3 \times 10^{-4}$ & $2.0 \times 10^{-4}$ \\
& 2 & 0.42 & $9.4 \times 10^{-4}$ & $0.7 \times 10^{-4}$ \\
& 3 & 0.04 & $-24.4 \times 10^{-4}$ & $12.2 \times 10^{-4}$ \\
\hline
\end{tabular}

This is a difficult numerical problem which can be solved by optimization methods. A solution to this problem is given in Dempster, Laird and Rubin (1977) who provide the E-M (ExpectationMaximization) algorithm. Table 1 gives the values of the estimated parameters for $K=2$ and $K=3$. The corresponding densities are shown in figure 2.

A careful examination of figure 2-b, shows that the adjustment of the empirical distribution for $K=2$ is quite good. The distribution of the data seems to be well approximated by a mixture of Gaussian distributions with a zero-mean and different variances.

$$
X_{i}-0.89 N\left(4 \times 10^{-4}, 1.2 \times 10^{-4}\right)+0.11 N\left(28.6 \times 10^{-4}, 7.3 \times 10^{-4}\right)
$$

When the number of components is $K=3$, an improvement can be seen in the adjustment of the empirical distribution (figure 2-d). Here, also, the distribution of the data is approximated by a mixture of Gaussian distributions with a zero-mean and different variances:

$$
\begin{aligned}
& X_{i}-0.54 N\left(-3.3 \times 10^{-4}, 2.010 \times 10^{-4}\right) \\
& +0.42 N\left(9.410 \times 10^{-4}, 0.0710 \times 10^{-4}\right) \\
& +0.04 N\left(-24.410 \times 10^{-4}, 12.210 \times 10^{-4}\right) .
\end{aligned}
$$

At this level, it is important to note that there is no strong statistical test 

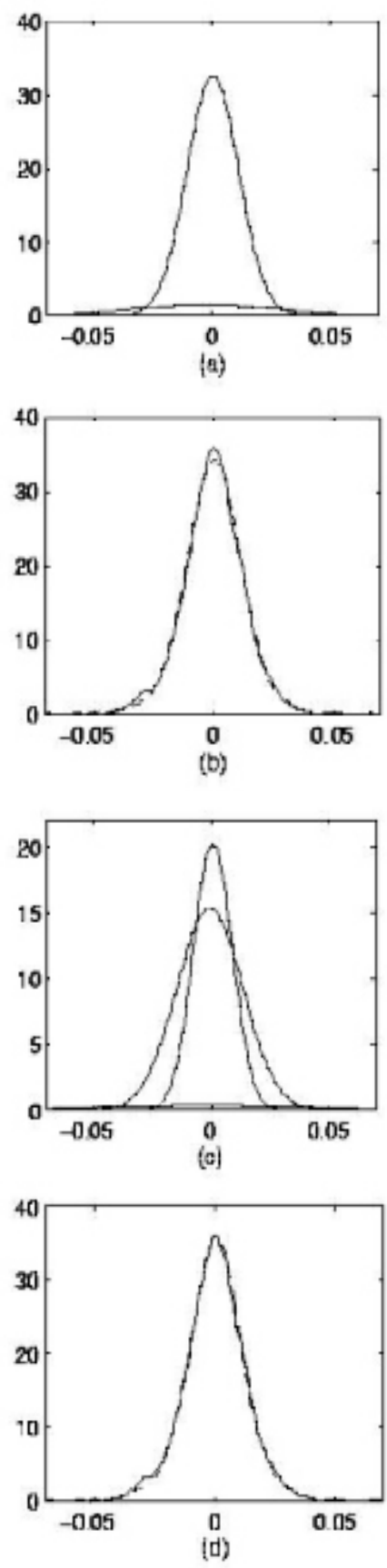

FIGURE 2.-Panels a through d. 
which allows to choose between the numbers of components 2 or 3 . However, it is possible to assert that the adjustment is good in both cases. The analysis and the explanations of the results by a specialist of a particular financial market having more information about the data would probably give more concrete sense to the numbers $K=2$ or $K=$ 3 and to the values of the estimated parameters.

Comparison of the estimated values of the kurtosis (3.97) and the skewness $(-0.32)$ with the theoretical values obtained with $K=2(3.17)$ and $K=3$ (3.73) reinforce the hypothesis of three different regimes. On the other hand, the skewness is not really informative since the estimated value $(-0.22)$ and the theoretical values obtained with $K=2$ $(-0.24)$ and $K=3(-0.17)$ are very close.

The first two populations are more represented (55\% and $41 \%)$. They can be thought of as representing two normal regimes: a stable period and a more turbulent period. The third population is less represented (environ 4\%) and exhibits a higher variance when compared to the other two populations. It characterizes more variability in the data and corresponds to a more turbulent period. It may refer to financial crisis, panics, crashes or mini-crashes, etc. These results seem to indicate the existence of three different periods in the pattern of the market volatility: a period of high volatility, a period of low volatility and a period of a stable volatility.

These three periods can be easily identified in the short or in a long run. Also, the three different scenarios of volatility in the financial market, may correspond to the different empirical distributions of assets returns which alternate through time. In fact, since market prices account for the probability of rare events, this induces an asymmetry in the distribution of returns and produces even fat and asymmetric tails with comparison to the returns of a normal distribution. A fat tail might correspond to the probability of a crash in the financial market or the probability of a takeover. When the market expects a sudden fall, the probability of a crash or a mini-crash is included in the distribution of asset prices. This probability may correspond to a very bad news announcement, liquidity shocks, banking problems, speculative bubbles, market structure deficiencies, asymmetric information, etc. The above reasons might explain the shapes of the empirical distributions observed in financial markets and the existence of the three regimes. The empirical distribution seems to be a superposition of three different normal distributions: one representing the normal regime corresponding to the central distribution and two other distributions corresponding to 
the tails of the empirical distribution. The first may represent the probabilities of crashes or sudden falls in the assets prices. The other tail may correspond to the probabilities of takeovers in the stock market. Hence, to each of the observed regimes, a normal distribution can be associated. The superposition of these distributions gives rise to the observed empirical distribution. The main question at this level is the following:

Can the periods of high risk be identified independently of the theoretical distribution used and the measures of risk?

If an algorithm of detection is proposed, then it would be possible to identify the periods of high volatility, corresponding to market crisis and panics. Contrary to the previous literature, we are able to provide a method which allows one to detect the changes in the regimes in the market place. Now, we provide an answer to this issue and apply our method to the CAC 40 index.

\section{Detection of changes in the distribution of the returns}

The previous analysis shows that the general method of decomposition allows the standard distributions to be replaced by any distribution from within a wide class. We have not imposed a particular assumption for the mixture of two or three distributions. A remark must be done at this level of our analysis: the data corresponds to a time-series and not to a sample in which the indexes are interchangeable. If we look to a part of this time-series (figure $3-a)$ comprising 400 observations, it is clear that the time-series is not stationary: the distribution of the data is not independent of time. In fact, the mean of this sub-sample seems to be zero but the variance can vary sometimes suddenly. For example, in the region 70-90, the dispersion of the data is more important than for the other data in the sub-sample. The time-series can not be modeled as a stationary process but rather as a stationary process with segments. Some abrupt changes affect the variance of the time-series at random times, but the distribution of the data does not vary between two successive sudden changes. We propose a model for detecting the sudden changes and which locating their positions. Let $X_{1}, \ldots, X_{n}$ be a sequence of observations. This sequence is composed of an unknown number of segments for which the distribution does not vary. Let $c_{1}, \ldots$, $c_{L-1}$ be the instants corresponding to abrupt changes. Let $c_{0}=0$ and $c_{L}$ $=n$. Let's denote by $X_{\ell}$ the vector of observations from the segment $\ell$ : 


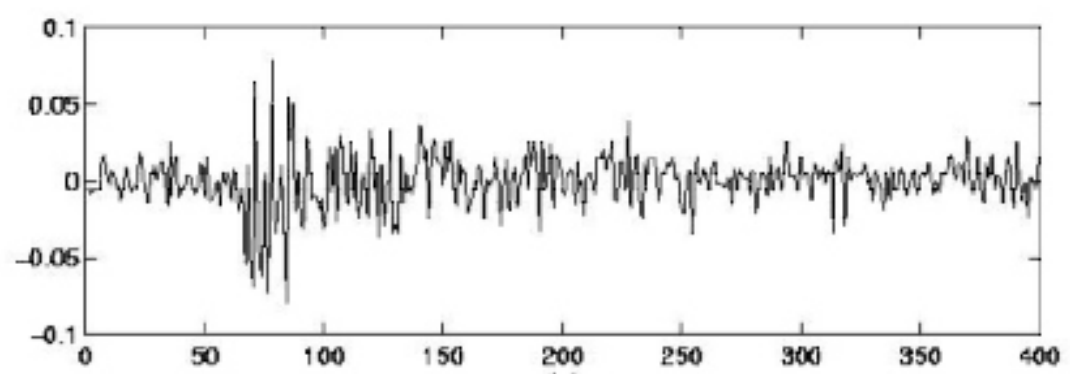

(a)

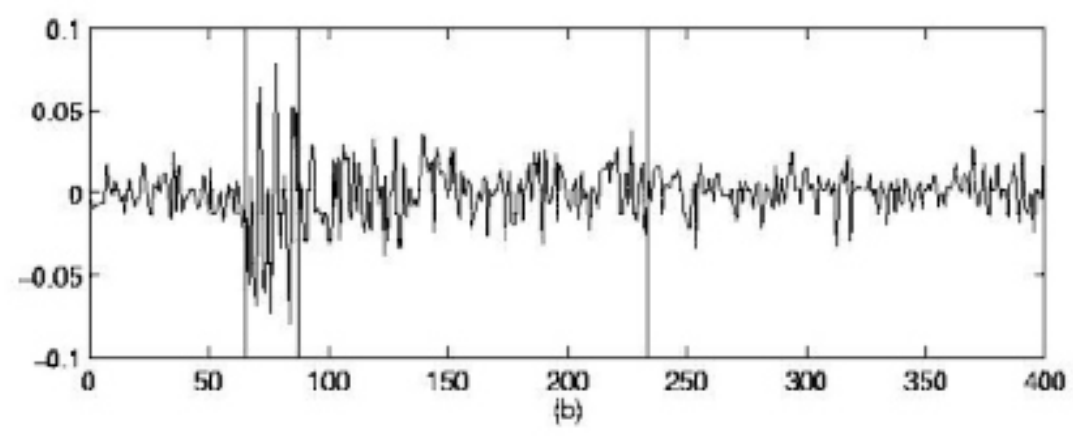

FIGURE 3.-Panels a and $\mathrm{b}$.

$$
X_{t}=\left(X_{c_{t-1}+1}, \ldots, X_{c_{t}}\right)
$$

For any $\ell>0$, let $\theta_{\boldsymbol{I}}=\left(\mu_{\boldsymbol{S}}, \sigma_{\boldsymbol{I}}^{2}\right)$, where $\mu_{\ell}$ is the mean of $X_{i}$ and $\sigma_{\boldsymbol{I}}^{2}$ is the variance of $X_{i}$ in the segment $\ell$, i.e; for $c_{\ell-1}+1 \leq i \leq c_{\ell}$.

When the form (configuration) of the abrupt changes $c=\left(c_{0}, \ldots, c_{L}\right)$ is given, $\theta_{\ell}$ can be estimated in the segment $\ell$ by minimizing the contrast function $U\left(X_{\ell}, \theta_{\ell}\right)$ defined by:

$$
U\left(X_{i}, \theta_{i}\right)=\frac{1}{\sigma_{i}^{2}} \sum_{i=c_{L-1}+1}^{\varepsilon_{Q}}\left(X_{i}-m_{i}\right)^{2}+n_{i} \log \sigma_{I}^{2},
$$

where $n_{\ell}=c_{\ell}-c_{\ell-I}$ is the length of the segment $\ell$.

This gives the empirical estimations of the mean and the variance: 


$$
\begin{gathered}
\hat{m}_{i}=\frac{1}{n_{i}} \sum_{i=\varkappa_{l-1}+1}^{c_{q}} X_{i}, \\
\hat{\sigma}_{i}^{2}=\frac{1}{n_{i}} \sum_{i=c_{t-1}+1}^{c_{l}} X_{i}^{2}-\hat{m}_{i}^{2} .
\end{gathered}
$$

When $c$ is unknown but the information regarding the number of segment $L$ is given, $c$ and $\theta=\left(\theta_{1}, \ldots, \theta_{L}\right)$ are estimated by minimizing the contrast function $V(c, \theta)$ defined by:

$$
\begin{gathered}
V(c, \theta)=\sum_{i=1}^{L} U\left(X_{i}, \theta_{i}\right) \\
=\sum_{i=1}^{L}\left(\frac{1}{\sigma_{i}^{2}} \sum_{i=c_{t-1}+1}^{c_{l}}\left(X_{i}-m_{i}\right)^{2}+n_{i} \log \sigma_{i}^{2}\right) .
\end{gathered}
$$

It must be noted that the number of abrupt changes is rarely known. However, we can estimate the configuration of the changes $c$ and the number of segments $L$ using the penalized minimum contrast estimator $(\hat{c}, \hat{\theta}, \hat{L})$ that is obtained by minimizing the function $W$ defined as:

$$
\begin{gathered}
W(c, \theta, L)=\sum_{i=1}^{L} U\left(X_{i}, \theta_{i}\right)+\beta L \\
=\sum_{l=1}^{L}\left(\frac{1}{\sigma_{l}^{2}} \sum_{i=\tau_{t-1}+1}^{r_{t}}\left(X_{1}-m_{l}\right)^{2}+n_{l} \log \sigma_{l}^{2}\right)+\beta L,
\end{gathered}
$$

where $\beta$ is a positive parameter. The procedure is intuitively simple: the adjustment criteria must be compensated for in a way such that the oversegmentation be penalized. However, the compensation must not be very important in a way such that it over-estimates the number of segments. In practice, the parameter $\beta$ must be fixed to some arbitrary value. When the parameter $\beta$ is chosen to be very large, only the more significant abrupt changes are detected. However, a small value of $\beta$ produces a high number of the estimated changes. Therefore, a trade-off 
TABLE 2. Estimation of the Length and the Parameters in the Different Segments Obtained with the Algorithm of Detection of Changes

\begin{tabular}{lrrr}
\hline segment $\ell$ & $\hat{n}_{\boldsymbol{z}}$ & \multicolumn{1}{c}{$\hat{m}_{\boldsymbol{z}}$} & \multicolumn{1}{c}{$\hat{\sigma}_{\boldsymbol{I}}^{2}$} \\
\hline 1 & 65 & $-11.1 \times 10^{-4}$ & $0.9 \times 10^{4}$ \\
2 & 22 & $-109.6 \times 10^{-4}$ & $20.7 \times 10^{4}$ \\
3 & 146 & $8.9 \times 10^{-4}$ & $3.0 \times 10^{4}$ \\
4 & 167 & $16.7 \times 10^{-4}$ & $1.1 \times 10^{4}$ \\
\hline
\end{tabular}

must be done, i.e, we have to select a value of $\beta$ which offers a reasonable level of resolution in the segmentation. ${ }^{2}$ Hence, we can show under some general hypothesis that the form (configuration) of the estimated abrupt changes converges to the true configuration of the changes when the number of observations $n$ increases. We can show also that the estimated parameters converge to the true parameters. When the changes affect the mean of the process, Lavielle and Moulines (1998) also derived the limiting distribution of the estimate of the change-point instants. The main theoretical results are presented in the Appendix. The segmentation proposed in figure 3-b is obtained with a value of $\beta=20$. It reveals four segments four which the values of the estimated parameters are given in table 2 .

We can see that there are no sudden changes in the mean return since the mean is often very close to zero. However, we detect three regimes in the variances of returns. The first regime corresponds to the first and the fourth interval on figure 3-b. It may refer to the normal regime of the volatility in the market place. This refers to stable periods. The second regime corresponds to the third interval. It shows more volatility than the normal regime and corresponds to turbulent periods. The third one corresponds to the second interval. It is over a very short interval and corresponds to chocks in the volatility. It is interesting to note that the results given by this segmentation method are exactly in accordance with those presented in the previous section. The time-series of returns seem to fit well the modeling by an alternance of three regimes. The passage from one regime to another is done in a stochastic way at random instants of time. For each of these three regimes, the distribution of the returns follow a Gaussian law with a zero mean.

2. Some practical considerations for the choice of $\beta$ are proposed by Lavielle (1998). 
Hence, we are in the presence of a mixture of three distributions with different variances.

It is important to note that in the segmentation method, we do not impose the number of mixing distributions. We find three regimes that give support to our early decomposition.

Now, a comparison is possible between our findings and previous work in this area.In the financial literature, the EM algorithm has been applied to our knowledge by Melick and Thomas (1997) for commodity prices, Lekkos (1999) for interest rate data and Demos and Sentana (1998) among others for factor models. Some financial institutions use the algorithm to handle the problems of missing data. The first study finds that the distribution of return fits well a mixture of three distributions. The second study assumes that the distribution of return fits well a mixture of two rather than three distributions. Our analysis applies the EM algorithm to stock returns using a new data set. Our first method, based on parametric and non-parametric approaches, show that there is a good fit of the empirical distribution using two or three normal distributions. In both cases, the fit is quite good. It is important to note that none of the above studies uses a stationary process with segments. Our model allows to detect systematically the sudden changes in the returns and locates their positions. In this model, the configuration of the changes and the number of segments are not known and can be estimated using a penalized minimum contrast estimator. We have shown somewhere how under general hypothesis the configuration of the estimated abrupt changes converges to the true configuration of the changes and that the estimated parameters converge to the true parameters. We have also derived the limiting distribution of the estimate of the change-point instants.

By applying this method, we find three regimes in the variances of returns. The results confirm our findings using the parametric and nonparametric approaches.

While these approaches are based on the assumption that the returns are generated from two or three possible scenarios in the market place (stable periods and turbulent periods), our segmentation method is independent from any prior assumption.

The above studies do not use the segmentation method in the analysis of the distributions of returns. The application of the segmentation method allows to detect the changes in returns from more recent data. This information can be useful is portfolio decisions, asset allocation and risk management. In fact, the use of the more recent 
information in financial returns can provide signals to portfolio managers to take the appropriate decisions for the future.

\section{Possible applications of the method}

The above analysis can be applied to improve the techniques in portfolio selection and risk management. This analysis can also be related and applied to the pricing of derivative securities. An extensive empirical literature has documented the presence of anomalies in the Black-Scholes (1973) model, (hereafter B-S) and in the term structure of these anomalies. This is the case for the behavior of the volatility smile or the unconditional returns at different maturities. Theoretical efforts have focused mainly on two extensions of the Black-Scholes model by introducing jumps into the return process and allowing for a stochastic volatility.

Attempts in the finance literature at reconciling the theory with the data have mostly centered around two classes of models: jump-diffusion models and stochastic volatility models. This is because these models lead to returns distributions that exhibit both skewness and kurtosis. Hence, each class of models could be made consistent with observed degrees of deviation from the Black-Scholes model. The theoretical predictions of either class of models do not seem to be consistent with the observed term structures of these deviations. Empirical tests of Black-Scholes type models reveal several empirical biases mainly because of the distribution of the underlying asset prices that seems to be with fat tails, high peaks, non symmetric, etc.

Das and Sundaram (1999) employ commonly used versions of these two classes of models and find that these models exhibit some term structure patterns that are fundamentally inconsistent with those observed in the data. They conclude that neither class of models represents an adequate explanation of the empirical evidence. Our work provides an alternative approach to handle these problems. ${ }^{3}$

Since the empirical distributions of the underlying assets are rather skewed and asymmetric, the use of a mixture of distributions allows to approximate the empirical distribution. If the observed distribution is

3. For more details, the reader can refer to Das and Sundaram (1999) and the references therein. 
used then the biases in B-S would disappear.

Consider an out of the money call. If the underlying asset price rises sufficiently, then its value will be higher. It is as if the value of the out of the money call is a function of the right hand tail of distribution. The B-S model has a tendency to misprice out of the money calls.

Consider an out of the money put. If the underlying asset price falls sufficiently, then its value will be higher. It is as if the value of the put is a function of the left hand tail of the distribution. The fatter is the tail, the higher is the put price. The Black-Scholes model has a tendency to misprice out of the money puts. ${ }^{4}$

Since the put-call parity is independent of the models used for option pricing, it can be used to determine the biases for in the money options. When the call is out of the money, the put is in the money and viceversa. Hence, an out of the money call should exhibit the same bias as an in the money put and vice versa. However, a more rigorous derivation of an option pricing model is possible in the lines of BlackScholes.

Using the martingale approach, the study of Rachev and Ruschendorf (1995) reveals that one of the most important feature of their model is that in most cases the prices of options can be explicitly calculated. So it relatively easy to analyze the sensibilities of the derivatives in this context. Moreover, many distributions for the laws of the stock returns can be obtained in the limit. In particular, this result gives a valuation like in the Black and Scholes (1973) model but with an underlying asset price that can have fat tails, higher peaks in the center, non symmetric shape, etc. The price process $\left(S_{t}\right)$ verifies the following dynamics:

$$
S_{t}=S_{0} \exp \left[\left(\mu-1 / 2 \sigma^{2}\right) t Y+\sigma \sqrt{Y} d B_{t}\right]
$$

So, the underlying asset price change movements follow the stochastic differential equation:

$$
\frac{d S_{t}}{S_{t}}=\mu Y d t+\sigma \sqrt{Y} d B_{t}
$$

4. For further evidence on the biases in the Black and Scholes (1973) model, the reader can refer to Dumas, Fleming and Whaley (1998). 
where $\left(B_{t}\right) t$ is a standard Brownian option and $Y$ is a random positive variable independent of $\left(B_{t}\right) t$ with the following distribution:

$$
P\left[Y=y_{j}\right]=\theta_{j}, j \in 1,2,3 .
$$

Let us denote by:

$$
\begin{gathered}
a_{j}=\alpha y_{j}, \text { and } \sigma_{j}^{2}=\sigma^{2} y_{j}, \\
\alpha=\mu-1 / 2 \sigma^{2}, \\
X_{t}=\alpha t+\sigma B_{t} .
\end{gathered}
$$

If the times of prices changes were deterministic then $S_{t}=S_{0} e^{X_{7}}$. The logarithmic of price changes denoted by $Z_{t}$ is described by the stochastic differential equation:

$$
d Z_{T}=\alpha Y d t+\sigma \sqrt{Y} d B_{t}
$$

Within the general framework developed by Harrison and Kreps (1979), the risk neutral valuation under the minimal martingale measure $\hat{Q}$ is given by its final payoff discounted to the present at the riskless interest rate:

$$
V_{0}=e^{-r T} E_{D}\left[V_{T}\right]
$$

where the mathematical expectation $E_{\hat{\theta}}$ is taken with respect to the risk-neutral probability $\hat{Q}$.

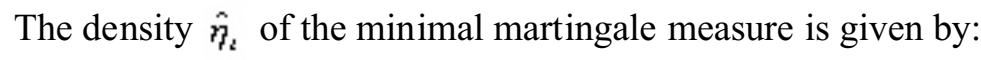

$$
E\left[\left.\frac{d \hat{Q}}{d P}\right|_{t}\right]=\exp \left(-\frac{1}{2} \frac{(\mu Y-r)^{2}}{Y \sigma^{2}}-\frac{(\mu Y-r)}{\sqrt{Y} \sigma} B_{t}\right)
$$

where $r$ refers to the riskless interest rate. One can also give another form for the evaluation formula which is the integral of the "classical" formula of the model of Black and Scholes (1973) with a drift $\mu y_{j}$ and 
a volatility $\sigma \sqrt{y_{j}}$ under the law of $Y$, that is:

$$
V_{0}=\sum_{j=1}^{3} \theta_{j} V_{E S}\left(y_{j}\right)
$$

For the case of standard options, it can be also noted that like in the Black and Scholes model, one has an option formula of the following form for the call: ${ }^{5}$

$$
C_{0}(K)=S_{0} \Phi_{z_{1}}\left(\log \left(\frac{K}{S_{0}}\right)-r T\right)-K e^{-r T} \Phi_{z_{2}}\left(\log \left(\frac{K}{S_{0}}\right)-r T\right),
$$

where

$$
\begin{aligned}
& Z_{1}=\frac{1}{2} \sigma^{2} Y+\sigma \sqrt{Y} \hat{B}_{T}, \\
& Z_{1}=-\frac{1}{2} \sigma^{2} Y+\sigma \sqrt{Y} \hat{B}_{T},
\end{aligned}
$$

and $\left(\hat{B}_{t}\right)_{t}$ is a Brownian motion under $\hat{Q}, K$ is the strike price and $\Phi$ is the cumulative inverse function of the normal distribution.

For the put, we have:

$$
P_{0}(K)=-S_{0} N_{Z_{1}}\left(\log \left(\frac{K}{S_{0}}\right)-r T\right)+K e^{-r T} N Z_{2}\left(\log \left(\frac{K}{S_{0}}\right)-r T\right),
$$

where $N$ is the cumulative function of the normal distribution. ${ }^{6}$

5. For the call, $C_{0}(K)=S_{0} \Phi(a, n, p)-K(1+r)^{-n} \Phi\left(a, n, p^{\prime}\right)$ in discrete time and in continuous time: $C_{0}(K)=S_{0} \Phi\left(-d_{1}\right)-K e^{-r t} \Phi\left(-d_{2}\right)$ where:

$$
d_{1}=\frac{\left[\log \frac{S_{0}}{K}+\left(r+\frac{\sigma^{2}}{2} T\right)\right]}{\sigma \sqrt{T}},
$$

and

$$
d_{2}=d_{1}-\sigma \sqrt{T} \text {. }
$$

6. The observed kurtosis in the distribution of stock price changes may be caused by the fact that returns follow a finite mixture of normal distributions. From the theorem 4.1 of 
The main difference between the option pricing model derived from the mixture-distribution and the standard Black-Scholes is that it can be more easily calibrated to the market. In fact, this model corresponds to a sum of Black-Scholes prices weighted by specific coefficients and shows more "degrees of freedom" than the standard Black-Scholes model. This has the potential to explain market prices because of the number of parameters used in the calibration which correspond to more degrees of freedom.

On the one hand, this model can appear as a special case of the models in Rachev-Ruschendorf (1995). While these authors do not provide a way to estimate the weighting coefficients in an explicit way, our approach offers a method to estimate these coefficients. This can be done using the EM algorithm.

Besides, in their paper, Rachev-Ruscendorf (1995) provide option pricing models in the presence of two randomizations: a randomization of the number of price changes and a randomization of the ups and downs in the price process. However, they do not provide statistical "tools" that allow the detection of the abrupt changes in market prices. Our segmentation method can be applied to their analysis to facilitate the empirical tests of their models.

While Ritchy (1990) and Rachev and Ruschendorf (1995) do not provide a way to estimate the weighting coefficients in an explicit way, our approaches offer complementary methods to estimate these coefficients from market data. This can be done using the EM algorithm or the segmentation method allowing their models to be used in real time by market participants. In fact, their models can be calibrated to

Rachev-Ruschendorf (1995), one deduces that the distribution of $Z_{t}, Z_{1, t}, Z_{2, t}$ are mixtures of normal distributions, i.e. the characteristic function $\phi z_{t}(u)=E\left[e^{i u Z t}\right]$ has the form:

$$
g z_{r}(u)=\int_{0}^{+\infty} \exp \left[t\left(i \alpha y u-\frac{\sigma_{u}^{2} y u^{2}}{2}\right)\right] d F_{r}(y) .
$$

So it is given by:

$$
g z_{i}(u)=\sum_{1=1}^{1} \theta_{1} \exp \left[t\left(i a_{1} u-\frac{\sigma_{1}^{2}}{2} u^{2}\right)\right] .
$$

Its density function is given by:

$$
f z_{r}(z)=\frac{2}{\sqrt{2 \pi t}} \exp \left[-\frac{(z \alpha y)^{2}}{2 y t \sigma^{2}}\right] d F_{r}(y)
$$


market data using our methods. We plan to test the class of models in Rachev and Ruschendorf (1995) and in Bellalah and Prigent (2002) using the above technology.

\section{Conclusion}

Several biases are reported in the empirical tests of capital asset pricing models and option pricing models, most of which are based on the simplifying assumptions regarding the distributions of financial asset prices. The normal assumptions are commonly used in the derivation of capital asset pricing models and option models. This is also the case for most standard risk management "tools". In fact, the methods proposed in risk management by RiskMetrics and CreditMetrics (among others) are also subject to several critics regarding the underlying distributions of financial assets.

Since the empirical distributions contain all the available information in capital markets, and represent a good description of the complexity of financial systems, numerous studies suggest the use of the observed distributions rather than the complex theoretical distributions when pricing financial securities. In this spirit, several authors studied the distributions of financial assets returns using two methods: the first is based on the stochastic processes and the second on the empirical observations. The study of the empirical distributions is a subject which is highly relevant for financial research. Also, the detection and estimation of the features of nonstationarities in data sequences are critical points in conducting credible research that uses data for inference.

In this vein, this paper applies and presents some results regarding the characterization of data with parameterized and nonparametrized forms and the determination of the stability of distributions. It discusses the approaches that have been previously used in the study of financial data and proposes a "new" segmentation method for the detection of changes.

Since market participants feel that prices are likely to be drawn from a multimodal distribution corresponding to different events in the market place, these events are expected to generate the shape of the mixing distributions of returns. Several financial explanations are given for the observed shapes of empirical distributions of assets returns.

Using the assumption of a mixing distributions as in Melick and Thomas (1997) and Lekkos (1999), we propose a simple decomposition 
of empirical distributions as a mixture of some normal distributions. Two alternative methods are used. The first method is a nonparametric approach based on a kernel estimate. The second is a parametric estimation based on the maximum likelihood criteria and the Expectation Maximization algorithm. The two methods are applied to the CAC 40 index data at the Paris Bourse. Both approaches give the same results and support the decomposition whether we use inter day or intraday data. We find that a mix of two or three distributions give an exact fit to the observed data. We extend our analysis by using a segmentation method. An algorithm of detection is developed to detect changes in the parameters of the distributions. The method allows to detect systematically sudden changes and locates their position. The form of abrupt changes in the distribution are estimated by minimizing a contrast function.

In the application of our segmentation method to the CAC 40 index and stocks traded in the Paris Bourse, we detect three regimes in the market place corresponding to different variances. Since this segmentation methodology supports the findings of the parametric and non-parametric approaches, we can assert that the observed distribution is generated from three mixing distributions.

Our work based on the EM algorithm and the segmentation method gives similar results and confirm the mixing distributions. It can be used in several contexts in the study of different distributions of returns observed in financial markets.

In a different context, Melick and Thomas (1997) use a different methodology to obtain a three mixing distributions in commodity markets (for oil). However, they do not confirm their results by applying our segmentation method. Besides, they do not examine the detection of changes and their exact locations. The segmentation methodology proposed here is new and can be applied to other data sets or other data generating conditions in other financial markets. Our results have some pricing implications.

First, since our parametric and non-parametric approaches allow the characterization of the observed distributions of returns, these mixing distributions with their appropriate weights can be used as inputs in the computation of $\mathrm{VaR}$ models using the observed distributions rather than the standard normal assumptions.

Second, the EM method proposed here is useful in handling the problems of missing data and in the identification of the exact fit to the empirical distributions. 
Third, since our segmentation method allows to detect the abrupt changes and their exact location, this latter feature can be used in real time with intraday data to provide some useful information for the reallocation of portfolios by individuals and institutions. Fourth, we provide an option pricing model that can be used for the valuation of standard and exotic derivatives.

Our work provides an alternative to the existing models. We plan to apply our results using intraday data in options markets and their underlying assets markets.

\section{Appendix: Theoretical results regarding the penalized minimum contrast estimator}

We present in this section some of our theoretical results concerning the penalized minimum contrast estimator proposed in section 4 for estimating the change-point instants.

Let $\left(X_{1}, \ldots, X_{n}\right)$ be the vector of observations. In order to obtain some asymptotical results concerning the penalized minimum contrast estimator proposed in section 4 for estimating the change-point instants, we must assume that the length of the segments increase when $n$ goes to infinity. More precisely, denoting $c_{n}^{*}=\left(c_{n, 1}^{*}, \ldots, c_{n, 1-1}^{*}\right)$ the true unknown vector of change-point instants, we shall assume that:

$$
\begin{gathered}
\mathrm{H}_{0}^{1} \text { : There exists } \tau^{*}=\left(\tau_{1}^{*}, \tau_{2}^{*}, \ldots, \tau_{L-1}^{\prime}\right) \text { with } 0<\tau_{1}^{*}<\tau_{2}^{*}<\ldots, \tau_{L-1}^{\prime}<1 \text {, such } \\
\text { that, for any } 1 \leq \ell \leq L-1, \quad c_{n, \ell}^{\prime} / n \rightarrow \tau_{\ell}^{\prime} \text { when } n \rightarrow \infty .
\end{gathered}
$$

Hypothesis $\mathrm{H}_{0}^{1}$ means that all the segment's lengths increase with the same rate $n$. We shall consider also the following hypothesizes:

$$
\begin{aligned}
& \mathrm{H}_{0}^{2} \text { : For any } 1 \leq \ell \leq L-1, \theta_{\ell}^{*}=\left(\mu_{\ell}^{*}, \sigma_{\ell}^{* 2}\right) \text { belongs to a compact set } \Theta \\
& \text { of } \mathbb{R} \times \mathbb{R}^{+} \text {. } \\
& \mathrm{H}_{0}^{3} \text { : Let } \varepsilon_{i}=X_{i}-E X_{i} \text { and } \zeta_{i}=\left(X_{i}-E X_{i}\right)^{2}-\operatorname{Var} X_{i} \text {. Then, there exist } \\
& C_{1}>0, C_{2}>0 \text { and } 1 \leq \phi<2 \text {, such that, for any } t \geq 0 \text { and any } m \\
& >0 \text {, } \\
& E\left(\sum_{i=+1}^{r+\infty} E_{1}\right)^{2} \leq C_{1} m^{\phi}, \quad E\left(\sum_{i=+1}^{r+m}\right)^{2} \leq C_{2} m^{\phi}
\end{aligned}
$$

Hypothesis $\mathrm{H}_{0}^{3}$ is satisfied for a very wide class of random processes, such as weakly or strongly dependent processes. For example, $\mathrm{H}_{0}^{3}$ holds with $\phi=1$ if, between two changes, $\left(X_{i}\right)$ is an ARMA process. If, between two changes, 
$\left(X_{i}\right)$ is a strongly dependent stationary Gaussian process, then, there exists $0 \leq$ $a<1$ such that $E X_{0} X_{k}=O\left(k^{-\mathrm{a}}\right), \forall k \leq 0$, and $\mathrm{H}_{0}^{3}$ holds with $\phi=\max (2-a, 1) .{ }^{7}$ For any $L>0$, let $C_{n, L}=\left\{\left(c_{1}, c_{2}, \ldots, c_{L-1}\right) \in \mathbb{N}^{L-1}, 0<c_{1}<c_{2}<\ldots<c_{L-1}<n\right\}$. and $\Theta_{\mathrm{L}}$ $=\Theta^{L}$. When the number $L$ of segments is known, we have the following result:

\section{Theorem A.1}

Let $\left(\hat{c}_{n}, \hat{\theta}_{n}\right)$ be the estimate of $\left(c^{*}, \theta^{*}\right)$ defined as the solution of the following minimization problem:

$$
V_{n}\left(\hat{c}_{n}, \hat{\theta}_{n}\right) \leq V_{n}(c, \theta) \text { for all }(c, \theta) \in\left(C_{n, L}, \theta_{L}\right)
$$

where

$$
V_{\mathrm{n}}(c, \theta)=\sum_{2=1}^{L}\left(\frac{1}{\sigma_{2}^{2}} \sum_{2=\tau_{\mathrm{t}-1+1}}^{\mathrm{r}_{\mathrm{c}}}\left(X_{1}-m_{\mathrm{l}}\right)^{2}+\left(c_{2}-c_{2-1}\right) \log \sigma_{2}^{2}\right) .
$$

Then, under $\left(\mathrm{H}_{0}^{1}, \mathrm{H}_{0}^{2}, \mathrm{H}_{0}^{3}\right)$, for any $1 \leq \ell \leq L-1,\left(\hat{c}_{n, L} / n, \hat{\theta}_{n, 2}\right)$ converges in probability to $\left(\tau_{\ell}^{*}, \theta_{\ell}^{*}\right)$ when $n \rightarrow \infty$. More precisely:

$$
\hat{c}_{n, 2}-\hat{c}_{n, 2}^{\prime}=O_{n}(1)
$$

and

$$
\hat{\theta}_{n z}-\theta_{2}^{\prime}=O_{n}\left(n^{b n^{2-1}}\right)
$$

When the true number $L^{*}$ of segments is unknown, we assume that an upper bound $I$ of $L^{*}$ is known. Then, we have the following result:

\section{Theorem A.2}

Let $\left\{\beta_{n}\right\}$ be a positive sequence of real numbers such that:

$$
\frac{B_{n}}{n} \rightarrow \infty
$$

and

$$
\frac{B_{n}}{n^{\vdash-1}} \underset{n \rightarrow \infty}{\rightarrow \infty}
$$

Let $\left(\hat{c}_{n}, \hat{\theta}_{n}, \hat{L}_{n}\right)$ be the estimate of $\left(c^{*}, \theta^{*}, L^{*}\right)$ defined as the solution of the following minimization problem:

7. Fore more details, see Lavielle (1996). 


$$
\begin{gathered}
W_{n}\left(\hat{\tau}_{n}, \hat{\theta}_{n}, \hat{L}_{n}\right) \leq W_{n}(\tau, \theta, L), \\
\forall(\tau, \theta, L) \in C_{n, L} \times \theta_{L} \times\{1,2, \ldots, L\},
\end{gathered}
$$

where

$$
W_{n}(\tau, \theta, L)=V_{n}(c, \theta)+\beta_{n} L
$$

Then, under $\left(\mathrm{H}_{0}^{1}, \mathrm{H}_{0}^{2}, \mathrm{H}_{0}^{3}\right),\left(\hat{c}_{n}, \hat{\theta}_{n}, \hat{L}_{n}\right)$, converges in probability to $\left(\tau^{\prime}, \theta^{\prime}, I^{\prime}\right)$.

\section{References}

Ait-Sahalia, Y., and Lo, A.W. 1995. Nonparametric estimation of state-price densities implicit in financial asset prices. Working paper series. N 5351, NBER.

Ait-Sahalia, Y. 1996. Testing continuous-time models of the spot interest rate. Review of Financial Studies 9: 385-426.

Basford, K.E., and McLachlan, G.J. 1985. Likelihood estimation with normal mixture models. Appl. Statist 34: 282-289.

Basseville, M., and Nikiforov, N. 1993. The detection of abrupt changes: Theory and applications. Prentice-Hall: Information and System sciences series.

Bellalah, M. 1990. Quatre essais pour l'evaluation des options sur indices et sur contrats a terme d'indice. Doctorat de l'Universite de Paris-Dauphine.

Bellalah, M. 1999 a. The valuation of futures and commodity options with information costs. Journal of Futures Markets September.

Bellalah, M. 1999 b. Les biais des modeles d'options revisites. Revue Francaise de Gestion June: 94-100.

Bellalah, M. 2000 a. A Risk Management Approach to options trading on the Paris Bourse. Derivatives Strategy 5: 31-33.

Bellalah, M. 2000 b. Valuation of American CAC 40 index options and wildcard options. International Review of Economics and Finance.

Briys E.; Bellalah M.; et al. 1998. Options, futures and other exotics. John Wiley and Sons.

Bookstaber, R. M., and McDonald, J.B. 1987. A General distribution for describing security price returns. Journal of Business 60: 401-424.

Blattberg, R., and Gonedes, N. 1974. A comparison of the stable and student distributions as statistical models for stock prices. Journal of Business 47: 244-280

Black, F., and Scholes. M. 1973. The pricing of options and corporate liabilities. Journal of Political Economy 81: 637-659.

Bollerslev, T. 1986. Generalized autore gressive conditional heteroskedasticity. Journal of Econometrics 31: 307-327. 
Choudhury, P.S. 1996. Optional extras. Risk June: 23-25.

Cox, J.; Ross, S.; and Rubinstein, M. 1979. Option pricing: A simplified approach. Journal of Financial Economics 7: 229-263.

Das, S.R., and Sundaram, R.K. 1999. Of smiles and smirks: A term structure perspective. Journal of Financial and Quantitative Analysis 34: 211-240.

Demos, A., and Sentana, E. 1998. An EM algorithm for conditionally heteroskedastic factor models. Journal of Business and Economics Statistics 16: 357-361.

Dempster, A.; Laird, N.; and Rubin, D. 1977. Maximum-likelihood from incomplete data via the EM algorithm. J. R. Statist. Soc. B 39: 1-38.

Dimson, E., and Marsh, P. 1995. Capital requirements for securities firms Journal of Finance 50: 821-851.

Dumas, B.; Fleming J.; and Whaley, R. 1998. Implied volatility functions: Empirical tests. Journal of Finance 6: 2059-2106.

Engle, R.F. 1982. Autoregressive conditional heteroskedasticity with estimates of the variance of United Kingdom inflation. Econometrica 50: 987-1006.

Engle, R.F. 1987. Multivariate ARCH with factor structures-cointegration in variance. Working paper. San Diego: University of California.

Epps, T.W., and Epps, M.L. 1976. The stochastic dependence of security price changes and transaction volumes: Implications of the mixture-ofdistribution hypothesis. Econometrica 44: 305-321.

Everit, B.S., and Hand, D.J. 1981. Finite mixture distributions. Monographs in Applied Probability and Statistics. Chapman and Hall, London.

Fama, E. 1963. Mandelbrot and the stable paretian hypothesis. Journal of Business 36: 420-429.

Fama, E. 1965. The behavior of stock market prices. Journal of Business 38: 34-65.

Hinckley, D.V. 1970. Inference about the change-point in a sequence of random variables. Biometrika 57: 1-17.

Kon, S.J. 1984. Models of stock returns: A comparison. Journal of Finance 39: 147-165.

Lavielle, M. 1999. Detection of multiples changes in a sequence of dependent variables. Stochastic Processes and their Applications, in press.

Lavielle, M. 1998. Optimal segmentation of random processes. IEEE Trans. on Signal Processing 46: 1365-1373.

Lavielle, M., and Moulines, E. 1999. Least squares estimation of an unknown number of shifts in a time series. Journal of Time-Series Analysis in press.

Leroux, B.G. 1992. Consistant estimation of mixing distribution. Annals of Statistics 20: 1350-1360.

Lekkos, I. 1999. Distributional properties of spot and forward interest rates: USD, DEM, GBP and JPY. Journal of Fixed Income 35-54.

Longin, F. 1996. The asymptotic distribution of extreme stock market returns. Journal of Business.

Mandelbrot, B. 1963. The variation of certain speculative prices. Journal of 
Business 36: 394-419.

Melick, W., and Thomas, C.P. 1997. Recovering an asset's implied pdf from option prices: An application to crude oil during the Gulf Crisis. Journal of Financial and Quantitative Analysis 32: 91-115.

Press, S. 1972. Multivariate stable distributions. Journal of Multivariate Analysis 2: 444-462.

Redner, R.A. and Walker, H.F. 1984. Mixture densities, maximum likelihood and the EM algorithm. SIAM Review 26: 195-223.

Ritchey, R.J. 1990. Call option valuation for discrete normal mixtures. Journal of Financial Research 13: 285-296.

Ryden, T. 1994. Consistant and asymptotically normal parameter estimates for hidden Markov models. Annals of Statistics 22: 1884-1895.

Ryden, T.; Terasvirta, T.; and Asbrink, S. 1999. Stylized facts of daily return series and the hidden Markov model. Journal of Applied Econometrics. forthcoming.

Rachev, S., and Xin, H. 1993. Test on association of random variables in the domain of attraction of multivariate stable law. Prob and Math Stat 14: 125-141.

Rachev, S., and Ruschendorf, L. 1995. Models for option prices. Theory Probab Appl 39: 120-152.

Somoroditsky, G., and Taqqu, M. 1994. Stable non-Gaussian random processes: Stochastic models with infinite variance. Chapman and Hall, New York.

Tauchen, G., and Pitts, M. 1963. The price-variability-volume relationship on speculative Markets. Econometrica 51: 485-505.

Titterington, D.M.; Smith, A.F.M.; and Makov, U.E. 1985. Statistical analysis of finite mixture distributions. John Wiley, Chichester. 